\title{
Burnout, liderazgo y satisfacción laboral en el personal asistencial de un hospital de tercer nivel en Bogotá*
}

\author{
Burnout, Leadership and Job Satisfaction in Health \\ Professionals of a Third Level Hospital in Bogotá
}

\author{
Françoise Contreras Torres* \\ Universidad del Rosario \\ Leonardo Espinal G. \\ Policía Nacional de Colombia
}

Ana María Pachón E.

Consultora independiente

Javier González R. Universidad del Rosario

Recibido: 2 de marzo de 2012 Revisado: 22 de mayo de 2012 Aceptado: 8 de agosto de 2012

\section{Resumen}

El propósito de este estudio fue identificar la presencia del Síndrome de Burnout, los estilos de liderazgo y la satisfacción laboral de los profesionales asistenciales de un hospital de tercer nivel y observar su relación, considerando variables sociodemográficas. Para ello, se utilizó el MBI, el TAP y el Test de Satisfacción Laboral S20/23, respectivamente. De acuerdo con los resultados, se encontró baja presencia de Burnout y altos niveles de realización personal, a pesar de las condiciones laborales adversas precursoras del síndrome. Contrario a lo reportado en la literatura, las personas con el síndrome o en riesgo de padecerlo presentan alta satisfacción laboral, sustentada en su motivación intrínseca, lo cual constituye un factor protector. No se encontró relación directa entre liderazgo y Burnout, pero el liderazgo sí se relaciona con la satisfacción laboral a través de la motivación intrínseca. Por último, se confirma el papel mediador de las variables sociodemográficas.

Palabras clave: Síndrome de Burnout, satisfacción laboral, liderazgo.

Artículo de investigación. Es resultado de los estudios del Grupo de Investigación en Perdurabilidad Empresarial (GIPE), línea de investigación en Liderazgo, Universidad del Rosario, Bogotá. Los autores agradecen la colaboración del profesor Juan Carlos Espinoza Méndez.

** Correspondencia: Françoise Contreras T.; correo electrónico: francoise.contreras@urosario.edu.co. Dirección Postal: Universidad del Rosario, Cra. 5 No. 15 -37, of. 201, Bogotá, Colombia. 


\section{Abstract}

The aim of this study is identify the presence of burnout; leadership styles and job satisfaction of professionals in a third level hospital and see if these are related, considering sociodemographic variables. We used the Spanish version of MBI, the TAP and Job Satisfaction Test S20/23. According to the results, we found low presence of burnout and high levels of personal accomplishment, despite the adverse labor conditions, commonly precursors of the syndrome. Contrary to reported in the literature, people with the syndrome or significant risk, have high job satisfaction, based on intrinsic motivation, which constitute a protective factor. There was no direct relationship between leadership and burnout, but leadership is relates to job satisfaction through intrinsic motivation. The mediating role of sociodemographic variables is confirmed.

Keywords: Burnout Syndrome, job satisfaction, leadership.

\section{Introducción}

De acuerdo con varios estudios, el trabajador de la salud tiene una mayor probabilidad de experimentar altos niveles de estrés laboral y padecer sus efectos adversos, no solo por lo que implica el ejercicio de su profesión, sino por las condiciones propias del contexto y del sector. Por sus características, las instituciones hospitalarias contribuyen a generar estrés, al ser organizaciones estructuralmente divididas, con relaciones que suelen estar mediadas por jerarquías fuertemente demarcadas entre el personal médico especializado, las enfermeras y los auxiliares. Tal condición puede incrementar la existencia de conflictos en el interior de los grupos de trabajo, generando un estrés permanente que puede comprometer la calidad del desempeño de los profesionales en la organización (Chiang, Salazar y Núñez, 2007).

Como respuesta a este nivel permanente de estrés se produce el llamado "Síndrome de Burnout" o "Síndrome de quemarse por el trabajo", el cual se manifiesta en una reducción de la eficacia profesional (Maslach, Jackson y Leiter, 1996) y constituye una de las más importantes causas de deterioro de la salud en estos profesionales (Farrerofis y Calvo, 2008), afectando principalmente a las enfermeras. Estas se consideran como el grupo de mayor riesgo de padecer el síndrome (Chacón y Grau, 1997), debido principalmente a la sobrecarga laboral, la escasa autonomía para la toma de decisiones, el poco reconocimiento profesional y el contacto cotidiano con la enfermedad, lo cual es altamente estresante (Zalon, 1995).

Ahora bien, aunque existe evidencia de estudios previos, la conceptualización y desarrollo del Síndrome de Burnout se le atribuye a Freudenberger $(1974,1977)$, quien observó el deterioro de la calidad de la atención que prestaba el personal de salud y lo atribuyó a la existencia del síndrome. Este autor lo definió inicialmente como la sensación de agotamiento, decepción y pérdida de interés por la actividad laboral que surge en aquellas personas que se dedican a asistir a otros. El síndrome básicamente se caracteriza por tener tres dimensiones, a saber: 1 . Agotamiento emocional o sensación de desgaste físico y mental, en la que el individuo se siente con poca energía, como si estuviera llegando a su propio límite; 2. Despersonalización o alteración en las relaciones que se establecen con las personas a quienes asiste, haciendo que la relación se vuelva distante e indiferente; 3 . Falta de realización personal o sentimientos de insuficiencia, fracaso, baja autoestima y desmotivación (Maslach y Jackson, 1981; Maslach, 1993).

Recientemente, Cáceres (2006) lo definió como un síndrome clínico caracterizado por un estado de fatiga o frustración, el cual se produce por la dedicación a una causa que no produce el efecto esperado. Maslach, Schaufeli y Leiter (2001), por su parte, afirman que el síndrome se debe principalmente al desequilibrio que se da entre las demandas organizacionales y los recursos personales, generando fatiga emocional y despersonalización; 
esta última como estrategia de afrontamiento inadecuada. Algunos autores llaman a este proceso "un fracaso adaptativo", caracterizado por un agotamiento físico, emocional y mental, despersonalización, bajo logro y escasa realización personal laboral, produciendo trabajadores cínicos, apáticos, escépticos e intransigentes (Gil-Monte y Peiró, 1999; Moreno, Oliver y Aragoneses, 1991)

Como podría esperarse, el Síndrome de Burnout tiene importantes efectos sobre la salud, el bienestar de los trabajadores, la calidad de su desempeño, la evolución de los pacientes y los procesos organizacionales. Las personas que padecen el síndrome presentan mayor tendencia al ausentismo, intención de abandonar el cargo, una actitud negativa hacia sus labores y disminución de la calidad del desempeño. Lo anterior ejerce influencia sobre el incremento de la rotación de personal y el aumento de quejas por parte de los usuarios (Martin, 1990; Maslach \& Jackson, 1981; Maslach et ál., 2001). Por el contrario, y confirmando los resultados anteriores, los empleados que experimentan mayor satisfacción laboral tienen más probabilidad de ser productivos y permanecer en el trabajo (McNeese-Smith, 1997; Irvine y Evans, 1995).

Entre los aspectos laborales que inciden en la aparición del síndrome están los relacionados con el trabajo en sí mismo y el contexto, los cuales pueden actuar como desencadenantes; por ejemplo, características propias de la tarea, aspectos técnicos del rol profesional, relaciones interpersonales, habilidad para trabajar con otros, ambiente de trabajo, grado de influencia social y política entre otros (Regina, Cardoso y Oliveira, 2010). Asimismo, las características de la organización y su cultura pueden incrementar la probabilidad de padecer el síndrome; por ejemplo, se ha observado que los cambios frecuentes de las organizaciones de salud inciden en que las personas deban enfrentar nuevas demandas laborales sin la información o los recursos necesarios, lo que incrementa el estrés laboral (Cáceres, Villanueva, Méndez, García y Domínguez, 2009).

Otra característica de la organización que induce a padecer el Síndrome de Burnout es el clima or- ganizacional, el cual está mediado por los estilos de dirección y las practicas de liderazgo (Cuadra y Veloso, 2010). Al respecto se ha observado que los líderes que favorecen la generación de ambientes, en los que se percibe la integración, apoyo y buenas relaciones interpersonales, protegen y mitigan el efecto de los estresores cotidianos (McNeeseSmith, 1997; Martínez, 2010; Topa, Fernández y Palací, 2006); además, incrementan potencialmente la satisfacción de los empleados (Bono y Judge, 2003; Cuadra y Veloso, 2010). De esta forma, las prácticas de liderazgo pueden influir de manera indirecta sobre el bienestar del trabajador y, consecuentemente, sobre la satisfacción laboral que este experimente, a través de su incidencia sobre el clima organizacional y la consolidación de la cultura organizacional (Contreras et ál., 2009).

En términos generales se puede afirmar que los comportamientos de liderazgo orientados hacia las personas, en los que se da importancia a la satisfacción de los trabajadores, incrementa la cohesión, reduce los conflictos disfuncionales y favorece la calidad de las relaciones entre directivos y subordinados (Upenieks, 2003). Por el contrario, un liderazgo autocrático y autoritario suele generar climas organizacionales proclives a fomentar el surgimiento de factores de riesgo psicosocial y afectar la salud de los miembros de la organización (Peiró, 2004); en estos estilos de liderazgo, la escasa autonomía y la falta de información para desempeñar las actividades puede predisponer al Síndrome de Burnout (Cáceres, 2006).

Por el contrario, el estilo de liderazgo transformacional incrementa el nivel de satisfacción laboral (Dunham-Taylor, 2000) y disminuye los niveles de estrés y la incidencia del Burnout (Sosik y Godshalk, 2000). Al parecer, una característica de este estilo de liderazgo que incide en la satisfacción laboral es aquella que prioriza la motivación intrínseca sobre la extrínseca; es decir, el líder se preocupa porque las actividades que realizan las personas les resulten interesantes y les proporcionen autosatisfacción. En contraste, la motivación extrínseca (propia de los estilos transaccionales) se basa en recompensas tangibles, que no provienen de la satisfacción por la actividad sino de estímulos externos (Porter y Lawler, 1968). 
Los estilos de liderazgo afectan la satisfacción laboral, aspecto que constituye un indicador fiable tanto del bienestar (Peiró et ál., 2004) como de la calidad de la atención. Este aspecto es especialmente relevante en el ámbito de la salud, en el que el componente humano y la calidez resultan esenciales (Jiménez y Pérez, 1999). Asimismo, la satisfacción de los usuarios está relacionada con la calidad de la atención y la satisfacción laboral de los trabajadores (Bravo, Peiró y Rodríguez, 1996; Morana, 1987).

En resumen, varios estudios apoyan la relación entre estilos de liderazgo y satisfacción laboral (Chiok, 2001; Lucas, 1991), el nivel de estrés percibido (Bratt et ál., 2000) y la motivación hacia el trabajo. Esto, en conjunto, afecta el nivel de satisfacción del paciente y la calidad de la atención prestada (McNeese-Smith, 1995), incidiendo de manera importante en la evolución de este (Morana, 1987), interés último y central de las organizaciones de salud.

Actualmente existe un amplio reconocimiento de la relevancia que tiene el bienestar de las personas en la organización y cómo este depende en gran medida de las prácticas de liderazgo. En este orden de ideas, existe un amplio consenso académico respecto a que la efectividad del liderazgo no puede medirse solo por niveles de productividad, sino que debe evaluarse en términos del comportamiento ético del líder (Kreitner, 1995) y de su capacidad para contribuir a la salud y el bienestar de los trabajadores, en el cual la satisfacción constituye un aspecto especialmente relevante (Perió y Rodríguez, 2008).

Ahora bien, de acuerdo con varios estudios, la escasa satisfacción laboral predispone al Síndrome de Burnout. El aburrimiento y la pérdida de interés pueden ser desencadenantes importantes del síndrome, al unirse con altos niveles de estrés, características individuales, personalidad del individuo, sobrecarga de trabajo y características del ambiente en el que se trabaja, entre otras (Cáceres, 2006; Abrajan, Contreras y Montoya, 2009; Hegney, Plank y Parker, 2006).
Cabe aclarar que la satisfacción en el trabajo, entendida como la actitud del trabajador hacia la condición laboral (Bravo et ál., 1996; Smith, Kendall y Hulin, 1975) no puede atribuirse a una sola causa; esta obedece también a otras variables relacionadas con el trabajo, entre ellas el salario, la supervisión, las condiciones propias de la actividad y de la organización, las oportunidades profesionales, el reconocimiento de las habilidades, la evaluación justa del trabajo, las prácticas organizacionales, el trato equitativo y las relaciones sociales laborales gratificantes, entre muchas otras (Misener et ál., 1996).

No obstante, es importante anotar que la relación entre satisfacción laboral y el Síndrome de Burnout depende también de características individuales, ya que hace referencia a lo que las personas piensan y sienten respecto a su trabajo, involucrando emociones, sentimientos, conductas y experiencias personales (Cuadra y Veloso, 2007). Estas últimas, a su vez, estarán relacionadas con sus características sociodemográficas, porque suelen influir en tales creencias.

Entre los factores individuales que incrementan la posibilidad de padecer el síndrome están el recibir baja remuneración, ser más joven y no percibir apoyo de la organización (Martínez, 2010). Maslach y Jackson (1985), por su parte, encontraron una prevalencia mayor del síndrome en mujeres por encima de los cuarenta años, especialmente en la dimensión de fatiga emocional (Ballenger-Browning et ál., 2011). Aranda et ál. (2010) encontraron en el personal de salud una mayor incidencia, en un rango de edad entre 30 y 40 años, al relacionar el grado de formación y la antigüedad en la institución (de 1 a 5 años). En este estudio, los hombres que laboraban en turnos mixtos fueron más susceptibles a padecer el síndrome. Al parecer, las variables demográficas pueden variar de acuerdo con el tipo de población estudiada; por ejemplo, se obtuvieron resultados contrarios en el personal sanitario militar. El ser más joven fue la variable de mayor relación con la aparición del síndrome (Cáceres, 2006).

Respecto al sexo, al igual que en el estudio citado anteriormente, hubo mayor prevalencia del 
Síndrome de Burnout en las mujeres, caracterizado principalmente por la fatiga emocional, sobre todo en aquellas que trabajaban largas jornadas y atendían un mayor número de pacientes (Ballenger-Browning et ál., 2011). En los hombres, la característica del Burnout más relevante fue la despersonalización. Con respecto al estado civil, se ha observado que el tener una familia y tener hijos parece amortiguar los efectos adversos del estrés; por consiguiente, las personas solteras suelen ser más susceptibles a padecer el síndrome (Cairo, 2002).

No obstante, los resultados empíricos en los que se estudia la distribución del Burnout entre hombres y mujeres son inconsistentes, probablemente por la existencia de problemas metodológicos tales como desigualdad entre las muestras o el desconocimiento del efecto del estatus ocupacional/marital que explican, en algunos casos, estas diferencias. Todo ello hace difícil describir y comparar la incidencia y prevalencia del síndrome en diferentes poblaciones. Este estudio resulta interesante en cuanto aún no se conoce de manera suficiente el fenómeno del Burnout en Colombia, población cuyas características resultan muy particulares. Tal como lo afirma Benevides-Pereira (2002), dadas las características del síndrome es recomendable desarrollar estudios específicos para cada población.

Con base en lo anterior, se pretende en este estudio describir la presencia del Síndrome de Burnout, el liderazgo percibido y la satisfacción laboral en profesionales de la salud y determinar si existe relación entre ellas, utilizando las variables sociodemográficas como mediadoras. Se pretende también determinar el perfil de los participantes que se encuentran en mayor riesgo de padecer el síndrome.

\section{Metodología}

Para el trabajo metodológico se utilizó un diseño exposfacto retrospectivo de tipo descriptivo.

\section{Participantes}

La selección de participantes se realizó a través de un muestreo aleatorio estratificado por profesiones. Se seleccionaron 309 participantes, con edades comprendidas entre los 20 y los 58 años, de los cuales cerca del 70\% fueron mujeres. Los participantes pertenecían a distintas áreas de la salud: medicina, bacteriología, enfermería, fisioterapia, fonoaudiología, instrumentación quirúrgica, nutrición y dietética, odontología, optometría, psicología, química farmacéutica, salud ocupacional, terapia ocupacional y respiratoria. Se excluyeron aquellos trabajadores de la salud que no ejercían labores asistenciales en el hospital o que se encontraban realizando de manera exclusiva actividades administrativas.

\section{Instrumentos}

\section{Maslach Burnout Inventory (MBI) (Maslach \& Jackson 1981, 1986).}

Se utilizó la versión en español de Gil-Monte y Peiró (1997). Se trata de un cuestionario autoadministrado, constituido por 22 afirmaciones que indagan acerca de los sentimientos y actitudes del profesional en su trabajo y hacia los pacientes. El participante debe elegir entre seis alternativas de respuesta, aquella que más se ajusta a la forma que experimenta su trabajo. Dichas alternativas van desde nunca (cero) hasta todos los días (seis). El instrumento provee información respecto a las tres dimensiones del síndrome, a saber, primero, realización personal (sentimientos de autoeficacia y realización personal); segundo, agotamiento emocional (experiencia de estar exhausto emocionalmente por las demandas del trabajo), y por último, despersonalización (grado en que cada uno reconoce actitudes de frialdad y distanciamiento). El MBI ha demostrado tener buenas propiedades psicométricas, es decir, una alta consistencia interna y una fiabilidad cercana al 0,9 .

\section{Test de Adjetivos de Pitcher (PAT)} (Pitcher, 1997)

El test consta de 60 adjetivos a través de los cuales los individuos evalúan a su jefe o supervisor. 
La autora determinó por medio de este instrumento la existencia de tres estilos de liderazgo, los cuales denominó artesanos, tecnócratas y artistas (Pitcher, 1999). No obstante, al hacer la traducción del instrumento y aplicarla a población colombiana, se identificaron dos categorías en las que los adjetivos se distribuyeron de manera diferencial en cada una de ellas: la primera demarca características deseables en un líder (humano, equilibrado, orientado hacia las personas, reflexivo, colaborador, honesto, franco, brillante, racional, cálido, digno de confianza, detallista, generoso, realista, abierto, analítico, emprendedor, firme, cortés y razonable), y la segunda incluye todas aquellas características que de acuerdo con la literatura no son deseables (impredecible, difícil, inflexible, rígido, extremista, fastidioso y testarudo). Cabe aclarar que ninguno de los adjetivos obtuvo saturaciones altas en las dos categorías de manera simultánea (Contreras et ál., 2009). De acuerdo con estudios psicométricos posteriores, se puede afirmar que dicho instrumento resulta adecuado para evaluar estilos de liderazgo en población colombiana (Juárez, Contreras y Barbosa, 2010).

\section{Test de Satisfacción Laboral S20/23 (Meliá y Peiró, 1989)}

Este instrumento ha sido diseñado para obtener una evaluación de la satisfacción laboral. Consta de 23 ítems con 7 alternativas de respuesta que van desde muy insatisfecho (1) hasta muy satisfecho (7). De acuerdo con los autores, el instrumento presenta adeudados niveles de fiabilidad y validez tanto a nivel global como en la descripción de los 5 factores de satisfacción. Estos niveles son con la supervisión, con el ambiente físico, con las prestaciones recibidas, satisfacción intrínseca del trabajo y satisfacción con la participación (Meliá y Peiró, 1989).

\section{Procedimiento}

Para el desarrollo del procedimiento, Previo consentimiento, se hizo una aplicación colectiva de los instrumentos a grupos de aproximadamente veinte personas en las instalaciones del hospital. Cabe aclarar que la participación en el estudio fue completamente anónima. Para ello, se utilizaron sobres cerrados con los instrumentos, los cuales incluían un lápiz para su diligenciamiento. Una vez la persona terminaba, lo devolvía en el mismo sobre. Es importante resaltar que la participación fue completamente voluntaria y que el participante podía decidir retirarse del estudio en el momento en que lo considerara conveniente.

\section{Resultados}

Inicialmente, se describirá cada una de las variables estudiadas, esto es, la presencia de Burnout, la percepción que se tiene del liderazgo y el nivel de satisfacción de los trabajadores en la organización. A su vez, se incluirá la información sociodemográfica de los participantes para observar si las características de este tipo juegan un papel mediador en las variables anteriormente enunciadas. Posteriormente, se observará a través de la comparación entre grupos si existen diferencias significativas entre los grupos resultantes en el análisis respecto a las variables estudiadas, con el fin de inferir la posible relación entre ellas. Finalmente, con base en lo anterior se observará si es posible elaborar un perfil de las personas que se encuentran en mayor riesgo de padecer el Síndrome de Burnout.

\section{Caracterización demográfica}

A nivel descriptivo, la muestra estuvo conformada por 309 participantes $(66,8 \%$ mujeres y $33,2 \%$ hombres) con edades entre 20 y 58 años $(M=$ $36,86$ y $S D=7,99)$, teniendo la mayoría $(63,5 \%)$ edades entre los 25 y 40 años. En cuanto al estado civil, reportaron en su mayoría estar solteros $(40,3 \%)$, casados $(46,1 \%)$, sin hijos $(39 \%)$, con un hijo $(28,1 \%)$ o dos hijos (25,5\%). Todos los participantes tienen estudios superiores en ciencias de la salud, la mayoría cuenta solo con el pregrado (71,9\%) y el $26,8 \%$ restante tiene una especialización, aspecto más frecuente en profesiones como enfermería, bacteriología, fisioterapia, medicina y odontología.

Dada la relevancia de los aspectos laborales en el estudio del Síndrome de Burnout, se indagó res- 
pecto a las condiciones laborales de estos profesionales. De allí se encontró que del total de participantes, la mayoría $(72,3 \%)$ tiene un contrato de prestación de servicios, seguido por vinculación como funcionario de planta por Ley 100 de 1993, equivalente a un $22,3 \%$. También se evidenció que el $36,1 \%$ trabaja en otras instituciones, siendo esta práctica de mayor frecuencia en los trabajadores contratados por prestación de servicios $(29,7 \%)$, en contraste con los trabajadorres de planta.

La labor desempeñada en el hospital corresponde en su mayoría a trabajo asistencial de forma exclusiva $(86,1 \%)$ y solo el $13,9 \%$ desarrolla, además, labores administrativas. Al considerar el tipo de vinculación laboral se aprecia que los trabajadores por prestación de servicios y los de planta se dedican en su mayoría al trabajo asistencial exclusivamente. Por otro lado, los trabajadores tienen una antigüedad de entre 0 y 30 años de servicios $(M=7,04 S D=6,10)$, estando la mayoría en un rango de 0 a 5 años (55,2\%), seguido por los rangos de 6 a 10 años $(16,8 \%$ ) y 11 a 15 años $(15,2 \%)$. Al tener en cuenta el tipo de vinculación, es evidente que la mayoría de los trabajadores por prestación de servicios se ubican en el rango de 0 a 5 años de antigüedad. Los cuatro trabajadores con más de 20 años de labores están vinculados actualmente por prestación de servicios.

Respecto a la dedicación semanal a las actividades laborales se reportaron entre 10 y 64 horas semanales $(M=40,39, S D=8,10)$, siendo lo más frecuente entre 41 y 50 horas semanales $(61,9 \%)$. Al respecto existen diferencias significativas entre quienes están vinculados a otra institución y los que no, presentándose una dedicación menor en los primeros $(M=37,96, S D=8,53$ vs. $M=41,76$, $\mathrm{SD}=7,534)$.

\section{Caracterización del Síndrome de Burnout}

Aunque se observan personas cuyas puntuaciones en la escala indicarían signos del Síndrome de Burnout, la tendencia en general de padecerlo es baja (tabla 1). No obstante, al observar por ran- gos los puntajes obtenidos en la escala, se aprecia una fuerte presencia de agotamiento emocional y despersonalización (figura 1).

Tabla 1.

Estadísticos descriptivos de las subescalas del MBI

\begin{tabular}{lcccccc}
\hline \multicolumn{1}{c}{ Subescala MBI } & No. & Mín. & Máx. & Media & $\begin{array}{c}\text { Desviación } \\
\text { típica }\end{array}$ & $\begin{array}{c}\text { Máx. } \\
\text { posible }\end{array}$ \\
\hline $\begin{array}{l}\text { Agotamiento } \\
\text { emocional }\end{array}$ & 309 & 0 & 53 & 18,21 & 13,34 & 54 \\
$\begin{array}{l}\text { Realización } \\
\text { personal }\end{array}$ & 309 & 0 & 30 & 6,85 & 7,82 & 48 \\
$\begin{array}{l}\text { Despersonali- } \\
\text { zación }\end{array}$ & 309 & 0 & 48 & 42,73 & 6,49 & 30 \\
\hline
\end{tabular}

Fuente: autores.

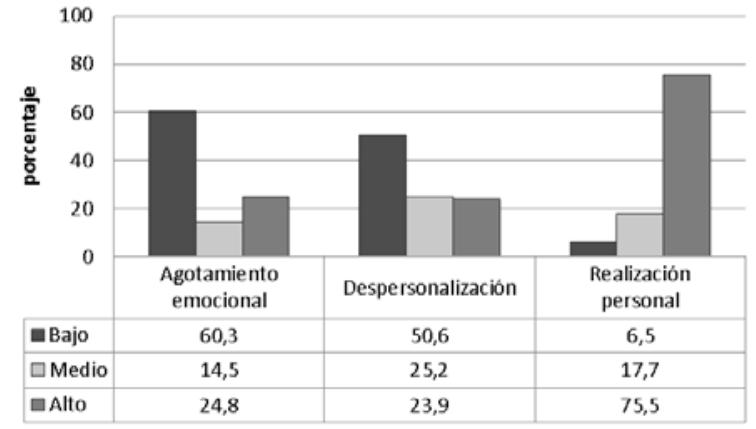

Figura 1. Distribución porcentual por niveles bajos, medios y altos en las subescalas del MBI.

Fuente: autores.

Se identificaron 7 participantes que presentan el síndrome, es decir, laboralmente se encuentran "quemados", esto es, altos niveles de agotamiento emocional y despersonalización, unido a un bajo nivel de realización personal (tabla 2), el cual representa el 2,3\% de la muestra. Con el propósito de hacer un análisis más detallado se establecieron 3 rangos de riesgo para el desarrollo de Burnout: el alto riesgo está dado por presentar altos niveles de agotamiento emocional y despersonalización, junto con un nivel medio de satisfacción (4,5\%); el riesgo bajo está configurado por bajos niveles de agotamiento emocional y despersonalización, junto con un nivel alto o medio de realización personal (37,4\%); el riesgo medio aplica para el porcentaje restante $(55,5 \%)$. Ahora bien, cuando se presenta alguna de las dos configuraciones siguientes, a saber, niveles medios de las tres dimensiones y niveles bajos de 
realización personal, se presenta un nivel alto en agotamiento emocional o en despersonalización.

Tabla 2.

Distribución porcentual de participantes según niveles en las subescalas del MBI

\begin{tabular}{lllll} 
& & \multicolumn{3}{c}{ Realización personal } \\
\hline $\begin{array}{c}\text { Agota- } \\
\text { miento } \\
\text { emocional }\end{array}$ & $\begin{array}{l}\text { Desperso- } \\
\text { nalización }\end{array}$ & Bajo & Medio & Alto \\
\hline \multirow{2}{*}{ Bajo } & Bajo & $1,3 \%$ & $4,2 \%$ & $33,3 \%$ \\
& Medio & $1,0 \%$ & $0,6 \%$ & $12,6 \%$ \\
& Alto & $0,6 \%$ & $3,2 \%$ & $3,6 \%$ \\
Media & Bajo & $0,3 \%$ & $0,3 \%$ & $6,5 \%$ \\
& Medio & $0,6 \%$ & $1,0 \%$ & $3,9 \%$ \\
& Alto & $0,0 \%$ & $0,6 \%$ & $1,3 \%$ \\
Alto & Bajo & $0,0 \%$ & $1,3 \%$ & $3,6 \%$ \\
& Medio & $0,3 \%$ & $1,9 \%$ & $3,2 \%$ \\
\hline Nonyyy & Alto & $2,3 \%$ & $4,5 \%$ & $7,8 \%$ \\
\hline
\end{tabular}

Nota: Se resalta el porcentaje de participantes con Burnout: 2,3\%, con un alto riesgo: $4,5 \%$; y un $4,2 \%$ y $33,3 \%$ en bajo riesgo de padecerlo.

Fuente: autores.

\section{Caracterización del liderazgo}

Para la caracterización, se calcularon los puntajes de las dimensiones del Test de Pitcher identificadas en el estudio de Contreras et ál. (2009), en el cual cada adjetivo se ponderó por el peso en cada factor; en consecuencia se obtuvo la suma y se transformó a puntuación $Z(M=0, S D=1)$ con el fin de hacer comparables las dos puntuaciones (tabla 3).
Tabla 3.

Estadísticas descriptivas para los estilos de liderazgo

\begin{tabular}{lccccc}
\hline $\begin{array}{l}\text { Estilos de } \\
\text { liderazgo }\end{array}$ & No. & Mín. & Máx. & $\begin{array}{c}\text { Me- } \\
\text { dia }\end{array}$ & $\begin{array}{c}\text { Des- } \\
\text { viación } \\
\text { típica }\end{array}$ \\
\hline $\begin{array}{l}\text { Líder 1 } \\
\text { (deseable) }\end{array}$ & 309 & 0,03 & 12,20 & 3,09 & 2,99 \\
$\begin{array}{l}\text { Líder 2 (no } \\
\text { deseable) }\end{array}$ & 309 & 0,00 & 3,30 & 0,56 & 0,51 \\
$\begin{array}{l}\text { Puntuación } \\
\text { Z: líder 1 } \\
\text { (deseable) }\end{array}$ & 309 & $-1,02$ & 3,05 & 0,00 & 1,00 \\
$\begin{array}{l}\text { Puntuación } \\
\text { Z: líder 2 (no } \\
\text { deseable) }\end{array}$ & 309 & $-1,07$ & 5,33 & 0,00 & 1,00 \\
\hline
\end{tabular}

Fuente: autores.

Ahora bien, con base en las puntuaciones estándar se establecieron dos grupos, los cuales son, bajo (inferior a la media) y alto (superior a la media). Finalmente, con el propósito de observar la tendencia en el estilo de liderazgo percibido, se clasificó a los sujetos y se encontró una marcada tendencia de no percepción de liderazgo $(43,4 \%)$, tal como se muestra en la tabla 4.

Tabla 4.

Clasificación y distribución por tipos de liderazgo percibido de acuerdo con los adjetivos del Test de Pitcher.

\begin{tabular}{lcccc}
\hline $\begin{array}{c}\text { Tipo de } \\
\text { liderazgo }\end{array}$ & $\begin{array}{c}\text { Líder } \\
\text { deseable }\end{array}$ & $\begin{array}{c}\text { Líder no } \\
\text { deseable }\end{array}$ & Frec. & $\%$ \\
\hline $\begin{array}{l}\text { No } \\
\text { liderazgo }\end{array}$ & Bajo & Bajo & 134 & 43,4 \\
$\begin{array}{l}\text { Liderazgo } \\
\text { no deseable }\end{array}$ & Bajo & Alto & 61 & 19,7 \\
$\begin{array}{l}\text { Liderazgo } \\
\text { ambivalente }\end{array}$ & Alto & Alto & 52 & 16,8 \\
$\begin{array}{l}\text { Liderazgo no } \\
\text { deseable }\end{array}$ & Alto & Bajo & 62 & 20,1 \\
\hline
\end{tabular}

Fuente: autores. 


\section{Caracterización de satisfacción laboral}

Se observa que en todas las dimensiones de satisfacción laboral el promedio de las puntuaciones obtenidas (rango de 1 al 7) es superior a la media, es decir, 3,5. En términos generales, el nivel de satisfacción es alto (5), siendo la satisfacción intrínseca la dimensión que más aporta a la satisfacción laboral, seguida por el ambiente físico y la supervisión. La dimensión que menos aporta a la satisfacción de los trabajadores es la relacionada con las prestaciones recibidas. En la figura 2 puede observarse la distribución de las dimensiones de satisfacción laboral de los participantes.

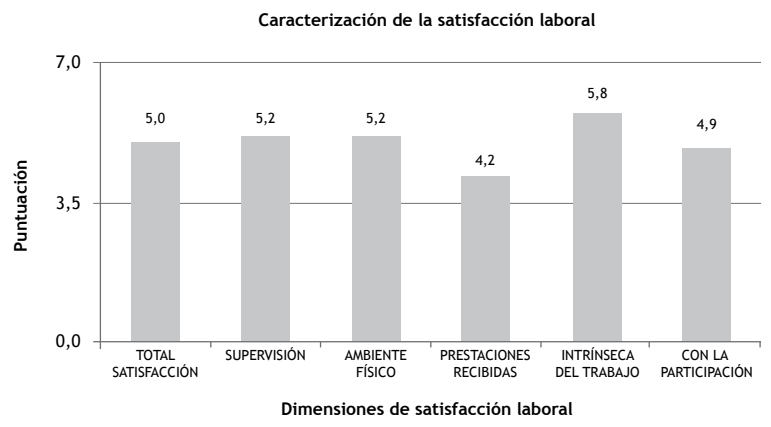

Figura 2. Distribución de las dimensiones de satisfacción laboral percibida entre los participantes

Fuente: autores.

\section{Correlaciones entre variables activas}

Se identificó una asociación entre la percepción del estilo de liderazgo y el Síndrome de Burnout (Chi2 $=18,726$, sig.: 028). En la tabla 5 se puede observar que los participantes que están en alto riesgo de desarrollar el Síndrome de Burnout o quienes ya lo desarrollaron perciben que la organización tiene un liderazgo no deseable o ambivalente. En cambio, en los niveles de riesgo bajo y medio no es clara la tendencia en la percepción de liderazgo.
Tabla 5.

Distribución porcentual en contingencia: liderazgo percibido vs. presencia del Síndrome de Burnout

\begin{tabular}{|c|c|c|c|c|c|}
\hline \multirow[b]{2}{*}{$\begin{array}{l}\text { Liderazgo } \\
\text { percibido }\end{array}$} & \multicolumn{4}{|c|}{ Burnout } & \multirow[b]{2}{*}{ Total } \\
\hline & $\begin{array}{c}\text { Riesgo } \\
\text { bajo }\end{array}$ & $\begin{array}{l}\text { Riesgo } \\
\text { medio }\end{array}$ & $\begin{array}{c}\text { Riesgo } \\
\text { alto }\end{array}$ & $\begin{array}{l}\text { Quema- } \\
\text { do }\end{array}$ & \\
\hline No liderazgo & $13,3 \%$ & $25,9 \%$ & $3,2 \%$ & $1,0 \%$ & $43,4 \%$ \\
\hline $\begin{array}{l}\text { Líder } \\
\text { no deseable }\end{array}$ & $5,8 \%$ & $12,9 \%$ & $0,3 \%$ & $0,6 \%$ & $19,7 \%$ \\
\hline Ambivalente & $8,7 \%$ & $6,8 \%$ & $0,6 \%$ & $0,6 \%$ & $16,8 \%$ \\
\hline $\begin{array}{l}\text { Líder } \\
\text { deseable }\end{array}$ & $9,7 \%$ & $10,0 \%$ & $0,3 \%$ & $0,0 \%$ & $20,1 \%$ \\
\hline Total & $37,5 \%$ & $55,7 \%$ & $4,5 \%$ & $2,3 \%$ & $100,0 \%$ \\
\hline
\end{tabular}

Fuente: autores.

Por otra parte, se encontraron diferencias estadísticamente significativas en todas las dimensiones de la satisfacción laboral. Al ser tenido en cuenta el nivel de riesgo del Síndrome de Burnout (tres dimensiones, cuando se tuvo en cuenta la percepción de los estilos de liderazgo, tabla 6), los efectos de la interacción de estas sobre la satisfacción no pudieron ser estimados, por la ausencia de normalidad estadística en la distribución de las mediciones de satisfacción laboral.

Tabla 6.

Prueba de comparación de grupos (Síndrome de Burnout y liderazgo) en la satisfacción laboral por medio de la prueba Kruskal-Wallis.

\begin{tabular}{lcccccc}
\hline \multirow{2}{*}{ Indicador } & \multicolumn{3}{c}{ Burnout } & \multicolumn{3}{c}{ Liderazgo } \\
\cline { 2 - 7 } & $\begin{array}{c}\text { Chi-cua- } \\
\text { drado }\end{array}$ & gl & $\begin{array}{c}\text { Sig. } \\
\text { asintót. }\end{array}$ & $\begin{array}{c}\text { Chi-cua- } \\
\text { drado }\end{array}$ & gl & $\begin{array}{c}\text { Sig. } \\
\text { asintót. }\end{array}$ \\
\hline Total & 15,949 & 3 & 0,001 & 17,824 & 3 & 0,000 \\
satisfacción & 19,108 & 3 & 0,000 & 33,031 & 3 & 0,000 \\
$\begin{array}{l}\text { Supervisión } \\
\text { Ambiente físico }\end{array}$ & 10,207 & 3 & 0,017 & 1,683 & 3 & 0,641 \\
$\begin{array}{l}\text { Prestaciones } \\
\text { recibidas }\end{array}$ & 21,112 & 3 & 0,000 & 7,671 & 3 & 0,053 \\
$\begin{array}{l}\text { Intrínseca del } \\
\text { trabajo }\end{array}$ & 10,899 & 3 & 0,012 & 7,211 & 3 & 0,065 \\
$\begin{array}{l}\text { Con la } \\
\text { participación }\end{array}$ & 13,375 & 3 & 0,004 & 16,420 & 3 & 0,001 \\
\hline
\end{tabular}

Fuente: autores.

Los participantes que tienen Síndrome de Burnout o tienen un alto riesgo de padecerlo presentan medias más altas en satisfacción. Respecto a la satisfacción laboral y liderazgo, se encontró 
mayor satisfacción con la supervisión y la participación en aquellos que perciben el liderazgo deseable y menor en los liderazgos no deseables, aspecto que impacta en la satisfacción general.

Ahora bien, aunque no fue posible estimar los efectos de interacción de niveles de Burnout y percepción de estilos de liderazgo, se aprecia que no hay ningún participante con el síndrome que observe un estilo de liderazgo deseable (figura 3). Asimismo, se observa que los participantes con alto riesgo de Burnout perciben ausencia de un estilo de liderazgo o un estilo deseable; en este último caso es importante revisar si existe o no un excesivo compromiso laboral.

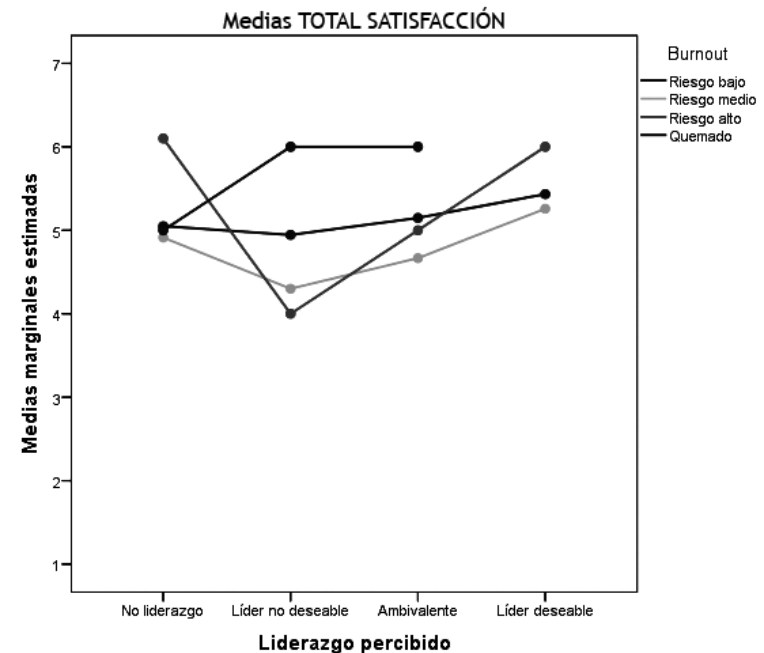

Figura 3. Medias del total de satisfacción laboral discriminadas por niveles de Burnout y estilo de liderazgo.

Fuente: autores.

En la tabla 7 puede observarse la comparación entre los grupos resultantes, de acuerdo con las variables estudiadas en conjunto.
Tabla 7.

Asociación entre variables de caracterización laboral y variables activas (Burnout y liderazgo)

\begin{tabular}{|c|c|c|c|c|c|c|}
\hline \multirow[t]{2}{*}{ Variables } & \multicolumn{2}{|c|}{$\begin{array}{l}\text { Nivel de } \\
\text { Burnout }\end{array}$} & \multicolumn{2}{|c|}{$\begin{array}{l}\text { Estilos de } \\
\text { liderazgo }\end{array}$} & \multicolumn{2}{|c|}{$\begin{array}{c}\text { Total de } \\
\text { satisfacción }\end{array}$} \\
\hline & $\mathrm{Chi}^{2}$ & Sig. & $\mathrm{Chi}^{2}$ & Sig. & $\mathrm{Chi}^{2}$ & Sig. \\
\hline $\begin{array}{l}\text { Nivel } \\
\text { académico }\end{array}$ & 26,17 & 0,002 & 15,95 & 0,068 & 23,63 & 0,001 \\
\hline Edad (rango) & 27,32 & 0,161 & 22,80 & 0,355 & 41,35 & 0,499 \\
\hline Género & 16,03 & 0,001 & 9,33 & 0,025 & 11,91 & 0,064 \\
\hline Estado civil & 6,88 & 0,961 & 21,31 & 0,127 & 170,04 & 0,000 \\
\hline Contratación & 21,64 & 0,010 & 4,38 & 0,884 & 14,01 & 0,728 \\
\hline Multiempleo & 9,78 & 0,020 & 24,57 & 0,000 & 5,63 & 0,465 \\
\hline $\begin{array}{l}\text { Exclusividad } \\
\text { asistencial }\end{array}$ & 2,30 & 0,513 & 11,03 & 0,012 & 10,28 & 0,113 \\
\hline Antigüedad & 8,48 & 0,903 & 19,06 & 0,211 & 37,62 & 0,160 \\
\hline $\begin{array}{l}\text { Dedicación } \\
\text { (h/s) }\end{array}$ & 36,30 & 0,198 & 49,18 & 0,015 & 72,96 & 0,122 \\
\hline Profesión & 64,56 & 0,006 & 76,72 & 0,000 & 140,47 & 0,000 \\
\hline $\begin{array}{l}\text { Horario } \\
\text { laboral }\end{array}$ & 48,08 & 0,001 & 26,85 & 0,176 & 87,64 & 0,000 \\
\hline $\begin{array}{l}\text { Jerarquía } \\
\text { laboral }\end{array}$ & 33,40 & 0,000 & 14,01 & 0,122 & 12,72 & 0,809 \\
\hline
\end{tabular}

Fuente: autores.

Por otro lado, los especialistas presentan en mayor proporción personas con Síndrome de Burnout. Estos representan un 1,3\% del 2,3\% con el síndrome, y un 3,6\% del 4,5\% con alto riesgo de "quemarse"; sin embargo, reportan mayor nivel de satisfacción laboral: del $27,1 \%$ de la muestra con este nivel de formación, el 13,1\% indica estar bastante satisfecho.

Adicionalmente, se muestran diferencias frente al sexo en el Burnout. Del 2,3\% de quemados, los hombres representan $1,9 \%$, y del $4,5 \%$ de alto riesgo de quemarse, los hombres representan el 2,6\%. No obstante, tanto hombres como mujeres reportan mayoritariamente la ausencia de liderazgo $(43,4 \%)$, por lo cual se observa una tendencia de los hombres a percibir liderazgo no deseable $(7,1 \%)$ y en las mujeres liderazgo deseable $(16,5 \%)$.

De igual manera, el tipo de contrato es un factor de riesgo para el síndrome. Todas las personas con el síndrome $(2,3 \%)$ presentan vinculación por prestación de servicios y representan más de la mitad de los que tienen alto riesgo de padecer el Burnout (2,6\% de 4,5\%). El multiempleo tam- 
bién es un factor de riesgo: quienes trabajan en otra institución son el 1,6\% del total de quemados $(2,3 \%)$ y el $2,6 \%$ de los que están en alto riesgo de Burnout (4,5\%). Tanto unos como otros, en su mayoría reportan ausencia de liderazgo, pero quienes laboran en otra institución reportan mayor percepción de liderazgo no deseable $(10,4 \%)$, en contraste con aquellos que reportan un uniempleo, los cuales perciben mayor liderazgo ambivalente $(12,9 \%)$ o deseable $(16,8 \%)$.

También se encontró que el total de trabajadores con el síndrome y la mayoría de los que tienen alto riesgo de quemarse $(4,2 \%$ de $4,5 \%)$ reportan dedicación exclusiva al trabajo asistencial; sin embargo, no hay significación estadística por el bajo porcentaje de trabajadores con doble condición de trabajo asistencial y administrativo $(13,9 \%)$. Quienes tienen doble rol tienen una tendencia a percibir un liderazgo no deseable $(4,9 \%)$, y los que tienen dedicación exclusiva asistencial perciben la ausencia de liderazgo (39,8\%).

Igualmente, la profesión es un factor de riesgo. Los profesionales en medicina representan el total de participantes que padecen el síndrome $(2,3 \%)$ y la mayoría de los que tienen alto riesgo de presentarlo $(3,9 \%$ de 4,5$)$. Cerca del $50 \%$ tanto de profesionales en medicina y enfermería como, en igual proporción, los auxiliares de enfermería reportan ausencia de liderazgo; la tercera parte de estos mismos grupos reportan un liderazgo no deseable, mientras que profesionales en odontología, terapias y bacteriología muestran una tendencia a percibir el liderazgo deseable.

Los trabajadores con el síndrome y con alto riesgo de padecerlo trabajan medio tiempo fijo, lo cual parece estar asociado con el multiempleo $\left(\mathrm{Chi}^{2}=\right.$ 25,926 , sig. $=0,001$ ); sin embargo, presentan niveles de satisfacción laboral similares a los que trabajan jornada completa en turnos fijos. Asimismo, quienes están en la primera línea de trabajo presentan mayor riesgo de padecer Burnout. Esta incluye a todos los quemados $(2,3 \%)$ y al $3,2 \%$ de los que tienen alto riesgo de quemarse $(4,5 \%)$; riesgo que comparten con los de segunda línea los supervisores, que representan el $1 \%$ de $4,5 \%$ con este nivel de riesgo.
Finalmente, al relacionar profesión, satisfacción laboral y Burnout, se encontró que los trabajadores con el síntoma o con alto riesgo de padecerlo son en su mayoría casi todos profesionales en medicina (19 de 21), que trabajan exclusivamente en trabajo asistencial (19 de 19), tienen multiempleo (13 de 19) y reportan ausencia de liderazgo (13 de 19). En general, este grupo de médicos reportan estar satisfechos laboralmente, aunque dicha satisfacción no se relaciona ni con las condiciones laborales ni con los estilos de liderazgo deseable.

\section{Discusión}

En este estudio se evidencian las características laborales propias del sector salud en Colombia, las cuales indiscutiblemente contribuyen en potencia al desarrollo del Síndrome de Burnout en los trabajadores, primordialmente en aquellos que realizan labores asistenciales, como ha sido documentado en varios estudios. Entre estas características están la inestabilidad laboral debida al tipo de contratación (72,3\% de los participantes están vinculados por prestación de servicios), reflejada en la permanencia de los trabajadores (algo más de la mitad llevan máximo 5 años en la institución) y la sobrecarga laboral, ya que los profesionales se ven en la necesidad de trabajar en otras lugares a la par con el hospital, trabajando así entre 40 y 50 horas a la semana $(61,9 \%$ de los participantes), lo cual es excesivo, más aún en labores que implican tanta responsabilidad como lo es la asistencia a pacientes, labor a la que se dedican exclusivamente casi todos los participantes en el estudio (el 86,1\%).

Asimismo, las instituciones de salud poseen características que predisponen al estrés laboral. Suelen ser organizaciones altamente reglamentadas y jerarquizadas en las que los cambios suelen ser frecuentes y exigen una rápida adaptación del personal para enfrentar las demandas laborales (Cáceres et ál., 2009). De esta manera, el ambiente y las condiciones están propicias para el desarrollo del síndrome, ya que estas podrían configurar desequilibrios entre las demandas organizacionales y los recursos personales en los procesos de adaptación a dichos cambios (Maslach et ál., 2001). 
No obstante, a pesar de las condiciones descritas anteriormente, se encontró, primero, un porcentaje bajo de personas con el Síndrome de Burnout $(2,3 \%)$ o con alto riesgo de padecerlo $(4,5 \%)$; segundo, una proporción importante de personas que se encuentran claramente en bajo riesgo, por tener bajos niveles de agotamiento emocional y despersonalización, acompañado de un nivel alto o medio de realización personal (37,4\%). Lo anterior denota la importancia de continuar estudiando poblaciones específicas como lo recomienda Benavides-Pereira (2002) y considerar aspectos particulares de las organizaciones tales como su cultura, categorías profesionales y características ocupacionales asociadas al síndrome, entre otras.

Una posible explicación de este hallazgo es que los niveles elevados de realización personal mitigan los efectos adversos del estrés y alcanzan a proteger a los profesionales de padecer el síndrome, o por lo menos, disminuye su probabilidad de aparición. Otra alternativa que valdría la pena estudiar es la capacidad adaptativa de estos profesionales para hacer frente a las demandas del ambiente bajo condiciones laborales adversas, pues no se ha dado el esperado fracaso adaptativo que conduce al Burnout y que ha sido ampliamente documentado (Gil-Monte y Peiró, 1999; Moreno et ál., 1991).

Asimismo, los participantes de este estudio experimentan una alta satisfacción laboral general, compuesta por elevados niveles de motivación intrínseca. Estas características pueden de forma combinada contribuir a proteger a los profesionales de padecer el síndrome. Se sugiere continuar investigando estas variables en particular, cuya relación parece ejercer un rol mediador ante la presencia del Síndrome de Burnout.

Ahora bien, frente a la evaluación que hacen los participantes sobre el estilo de liderazgo se observó una clara tendencia a no percibir un liderazgo por casi la mitad de los participantes $(43,4 \%)$. Las demás formas de liderazgo (deseable, no deseable y ambivalente) se distribuyeron de forma similar entre los participantes. Lo anterior puede estar asociado a los cambios frecuentes que se dan en este tipo de instituciones.
No obstante, la percepción que tienen los participantes sobre el liderazgo sí parece tener relación con la presencia de Burnout, pues aquellos profesionales que experimentan el síndrome no perciben, en ningún caso, un liderazgo deseable. Lo anterior coincide con lo que plantean Cuadra y Veloso (2010), que afirman que la predisposición al síndrome está mediada por los estilos de liderazgo, y que los líderes considerados deseables favorecen el bienestar de las personas, mitigando el efecto del estrés (McNeese-Smith, 1997; Martínez, 2010; Topa et ál., 2006; Upenieks, 2003). Sin embargo, entre aquellos que aún no presentan el síndrome pero están en alto riesgo se percibe o bien un liderazgo deseable o una ausencia de este, lo cual contradice lo encontrado en otros estudios en los que se afirma que un liderazgo favorable disminuye los niveles de estrés $y$, por ende, la incidencia del Burnout (Sosik y Godshalk, 2000).

Respecto a las dimensiones de satisfacción laboral, se observó que estas difieren de manera significativa de acuerdo con el nivel de Burnout que presenten los participantes. Curiosamente, las personas que tienen Burnout o tienen alto riesgo de padecerlo presentan niveles mayores de satisfacción laboral, lo cual puede explicarse por la alta deseabilidad social que suele acompañar el síndrome. Se recomiendan futuros estudios para dilucidar esta relación.

La motivación intrínseca que influye principalmente en la satisfacción laboral de los profesionales parece desarrollar sobre la forma en que se percibe al líder, esto es, que aquellos que perciben al líder como deseable suelen tener mayores niveles de satisfacción laboral en general y elevados niveles en las dimensiones de supervisión, motivación intrínseca del trabajo y satisfacción con la participación. Estos hallazgos también coinciden con lo planteado por otros autores: Bono y Judge (2003), Cuadra y Veloso (2010), DunhamTaylor (2000), Peiró et ál. (2004), quienes han encontrado que el liderazgo favorable incrementa potencialmente la satisfacción de los empleados. Estos resultados corroboran los hallados en otros estudios realizados con población colombiana, en los que se encontró que el estilo de liderazgo puede influir en el bienestar del trabajador $\mathrm{y}$, 
consecuentemente, en la satisfacción laboral que este experimente (Contreras et ál., 2009).

En este estudio se evidencio cómo el liderazgo centrado en las personas (deseable) se hace más evidente en la satisfacción laboral a través de la motivación intrínseca que en la extrínseca. Tal como lo planteó hace varias décadas Porter \& Lawler (1968), prueba de ello es que los factores externos asociados con la satisfacción (ambiente físico y las prestaciones recibidas) no muestran diferencias significativas entre las personas que perciben determinado tipo de liderazgo. Lo anterior denota, por una parte, el efecto diferencial que tienen los aspectos objetivos y subjetivos sobre la satisfacción laboral; y por otra, la importancia de los primeros como determinantes en la satisfacción laboral. Otro aspecto que vale la pena destacar es lo inadecuado que resulta atribuir la satisfacción laboral a una sola causa y la importancia de abordarla como un concepto complejo relacionado con diversas variables, tanto del entorno de trabajo como de características individuales, como lo señalan Misener et ál. (1996).

Por otra parte, los factores sociodemográficos sí constituyen variables mediadoras en el Síndrome de Burnout, la satisfacción laboral y la percepción de liderazgo. Al respecto, el nivel académico en esta población constituye un factor importante por considerar, pues los especialistas representaron mayor proporción de personas con Burnout o en alto riesgo de padecerlo; sin embargo, reportan mayor nivel de satisfacción laboral. Asimismo, el sexo es otro factor diferenciador: los hombres en este estudio presentan más Burnout que las mujeres, se encuentran en mayor riesgo de padecerlo y perciben un liderazgo no deseable, mientras que las mujeres están en menos riesgo y tienen mayor tendencia a percibir el liderazgo deseable. Estas diferencias pueden estar más asociadas al género que al sexo y puede sustentarse en los roles entre unos y otros, mediando las relaciones diferenciales que suelen darse entre hombres y mujeres en el contexto laboral.

Estos resultados confirman el papel mediador de las variables sociodemográficas, pero en forma distinta; es decir, en este estudio la edad no fue un factor asociado al Burnout, como lo hallaron otros autores (Martínez, 2010; Ballenger-Browning et ál., 2011; Aranda et ál., 2010); y contrario a lo encontrado por Maslach y Jackson (1985) y Ballenger-Browning et ál. (2011), la prevalencia fue mayor en los hombres que en las mujeres.

Asimismo, como se mencionó anteriormente, el tener una sobrecarga laboral predispone al síndrome (multiempleo). Esto se confirma al observar que la mitad de quienes lo padecen o están en alto riesgo de padecerlo, trabajan en distintos sitios. Estos resultados coinciden con los de Aranda et ál. (2010), que encontraron que las personas que trabajan en varios turnos suelen ser más susceptibles de padecer el síndrome. Dicha sobrecarga parece afectar negativamente la percepción que el individuo tiene sobre el estilo de liderazgo, pues tienden a percibirlo como no deseable. Por el contrario, las personas que no tienen sobrecarga laboral (trabajan en un solo sitio) perciben al líder como más deseable o, por lo menos, ambivalente.

Tal como aparece en la literatura, el desempeñar un trabajo puramente asistencial predispone al Burnout o pone al individuo en mayor riesgo de padecerlo. Según este estudio, el tipo de profesional de la salud que está más expuesto al síndrome es el médico (del total de personas con Burnout, todos son médicos, al igual que aquellos que tienen alto riesgo de padecerlo). Estos resultados contradicen los hallados por otros autores en los que se ha observado que el síndrome afecta principalmente a las enfermeras, considerándose el grupo de mayor riesgo (Chacón y Grau, 1997; Zalon, 1995). Habría que estudiar el efecto de las variables socioculturales que pueden tener algún efecto en estos resultados. De forma coherente, el personal de salud con más alto rango en la jerarquía o el que le sigue está en mayor riesgo de padecer el síndrome o tenerlo. Esto puede deberse a las diferencias que por profesiones presentan los individuos respecto a la percepción del liderazgo, aspecto que valdría la pena indagar.

De la misma manera, como lo plantea Regina et ál. (2010), en este estudio se encontró que, efectivamente, los aspectos laborales relaciona- 
dos con el trabajo en la salud, los factores contextuales (características propias del ejercicio de la profesión), particularmente la asistencia, los aspectos técnicos del rol profesional y el grado de influencia social pueden actuar como desencadenantes del síndrome.

Finalmente, el perfil del trabajador con Síndrome de Burnout o con alto riesgo de padecerlo se compone de las siguientes características: 1 . Ser profesional de medicina; 2 . estar dedicado exclusivamente al trabajo asistencial; 3. Trabajar en varios sitios; 4. Percibir que existe ausencia de liderazgo en su institución. Estas personas, como contraparte, reportan estar bastante satisfechas laboralmente, aspecto que requiere ser estudiado en profundidad con el fin de identificar cuál es la fuente de tal satisfacción, ya que según los datos encontrados, no están relacionados ni con las condiciones laborales ni con los estilos de liderazgo deseable.

Estos hallazgos cobran especial importancia si se considera que varios estudios han encontrado que la escasa satisfacción laboral predispone al Burnout al unirse a determinadas características personales, tales como la sobrecarga de trabajo y características del ambiente (Cáceres, 2006; Abrajan et ál., 2009; Hegney et ál., 2006), y si se tiene en cuenta que dicha relación puede afectar la calidad de la tensión en estos profesionales, lo cual en salud es una prioridad evidente.

\section{Referencias}

Abrajan, M. G., Contreras, J. M. y Montoya, S. (2009). Grado de satisfacción laboral y condiciones de trabajo: una exploración cualitativa. Enseñanza e Investigación en Psicología, 14(1), 105-118.

Aranda, C., Zárate, B., Pando M. y Sahún, J. E. (2010). Síndrome de Burnout, variables sociodemográficas y laborales en médicos especialistas del hospital Valentín Gómez Farias. Revista Colombiana de Salud Ocupacional, 1(1), 12-16. México.
Ballenger-Browning et ál. (2011). Predictors of burnout among military mental health providers. Military Medicine, 176(3), 253-260.

Benevides-Pereira, A. (2002). Burnout: quando o trabalho ameaça o bem estar do trabaihador. São Paulo: Casa do Psicólogo.

Bono, J. E. \& Judge, T. A. (2003). Self-concordance at work: toward understanding the motivational effects of transformational leaders. Academy of Management Journal, 46, 554-571.

Bratt, M. M., Broome, M., Kelber S. \& Lostocco, L. (2000). Influence of stress and nursing leadership on job satisfaction of pediatric intensive care unit nurses. American Journal of Critical Care, 9(5), 307-17.

Bravo, M. J., Peiró, J. M. y Rodríguez, I. (1996). Satisfacción laboral. En J. Peiró M. y F. Prieto (Eds.). Tratado de Psicología del Trabajo (pp. 347-394). Madrid: Síntesis.

Cáceres, G. (2006). Prevalencia del Síndrome de Burnout en personal sanitario militar (tesis doctoral). Madrid: Universidad Complutense de Madrid.

Cáceres, G., Villanueva, R., Méndez, J. R., García, M. V. y Domínguez, V. (2009). Prevalencia del Síndrome de Burnout en médicos y enfermeros de un hospital militar. Sanidad Militar, 65(2), 83-94.

Cairo H, (2002). Burnout o estrés laboral: un síndrome cada vez más expandido. Recuperado de www.mujeresdeempresa.com/realciones_ humanas/relaciones020402.sthml

Chacón, R. M. y Grau, J. (1997). Burnout en enfermeros que brindan atención a pacientes oncológicos en Cuba. Revista Cubana de Oncología, 13(2), 118-125.

Chiang M. M., Salazar, C. M. y Núñez, A. (2007). Clima organizacional y satisfacción laboral en un establecimiento de salud estatal: Hospital tipo 1. Theoria 16(2), 61-76. 
Chiok, J. (2001). Leadership behaviours: effects on job satisfaction, productivity and organizational commitment. Journal of Nursing Management, 9, 191-204.

Contreras, F., Barbosa, D., Juárez, F., Uribe, A. F. y Mejía, C. (2009). Estilos de liderazgo, clima organizacional y riesgos psicosociales en entidades del sector salud. Un estudio comparativo. Acta Colombiana de Psicología 12(2), 13-26.

Cuadra, A. y Veloso, C. (2007). Liderazgo, clima y satisfacción laboral. Revista Universum, 22(2), 40-56.

Cuadra, A. y Veloso, C. (2010). Grado de supervisión como variable moderadora entre liderazgo y satisfacción, motivación y clima organizacional. Revista Chilena de Ingeniería, 18(1), $15-25$.

Dunham-Taylor, J. (2000). Nurse executive transformational leadership found in participative organizations. Journal of Nursing Administration, 30(5), 241-50.

Farrerofis, L. y Calvo, F. (2008). Estudio descriptivo del Síndrome de Burnout en una muestra de profesionales de enfermería en el área sur de gran canaria. Ansiedad y Estrés, 14(1), 101-113.

Freudenberger, H. J. (1974). Staff Burn-out. Journal of Social Issues, 30, 159-165.

Freudenberger, H. J. (1977). Burn-out: the organizational menace. Trainning and Development Journal, 31, 26-27.

Gil-Monte, P. R. y Peiró, J. M. (1997). Desgaste psíquico en el trabajo: el síndrome de quemarse. Madrid, España: Síntesis.

Gil-Monte, P. R. y Peiró, J. M. (1999). Perspectivas teóricas y modelos interpretativos para el estudio del síndrome de quemarse por el trabajo. Anales de Psicología, 15(2), 261-268.
Hegney, D., Plank, A. \& Parker, V. (2006). Extrinsic and intrinsic work values: their impact on job satisfaction in nursing. Journal of Nursing Management, 14(2), 271-281.

Irvine, D. M. \& Evans, M. G. (1995). Job satisfaction and turnover among nurses: integrating research findings across studies. Nursing Research, 44, 246-252.

Jiménez, E. y Pérez, E. (1999). Satisfacción laboral de la enfermera. Revista de Enfermería, 7(3), 177-180.

Juárez, F., Contreras, F. y Barbosa, D. (2010). Propiedades psicométricas del test de adjetivos de Pitcher para la evaluación del liderazgo (inédito).

Kreitner, R. (1995). Management (6. ${ }^{\text {th }}$ ed.). Boston: Houghton Mifflin Company.

Lucas, M. D. (1991). Management style and staff nurse job satisfaction. Journal of Professional Nursing, 7(2), 119-125.

Martin, B. J. (1990). A successful approach to absenteeism. Nursing Management, 21, 45-48

Martínez, A. (2010, septiembre). El Síndrome de Burnout. Evolución conceptual y estado actual de la cuestión. Vivat Academia, 112. Recuperado de http://www.ucm.es/info/vivataca/numeros/n112/DATOSS.htm

Maslach, C. (1993). Burnout: a multidimensional perspective. En W. B. Schaufeli, C. Maslach y T. Marek (Ed.). Professional burnout (pp. 1932). Washington: Taylor and Francis.

Maslach, C. \& Jackson, S. E. (1981). The measurement of experienced burnout. Journal of Occupational Behavior, 2, 99-113.

Maslach, C. \& Jackson, S. (1985). The role of sex and family variables in Burnout. Sex roles, 12(7-8), 837-851. 
Maslach, C. \& Jackson, S. E. (1986). Maslach Burnout Inventory. Palo Alto, CA: Consulting Psychlogists Press.

Maslach, C., Jackson, S. E. \& Leiter, M. P. (1996). Maslach Burnout Inventory Manual (3. ${ }^{\text {rd }}$ ed.). Palo Alto: Consulting Psychologists Press.

Maslach, C., Schaufeli, W. B. \& Leiter, M. P. (2001). Job burnout. Annual Review of Psychology, 52, 397-422.

McNeese-Smith, D. K. (1995). Job satisfaction, productivity and organisational commitment, the result of leadership. Journal of Nursing Administration, 25, 17-26.

McNeese-Smith, D. K. (1997). The influence of manager behavior on nurses' job satisfaction, productivity and commitment. Journal of Nursing Administration, 27, 47-55.

Meliá, J. L. y Peiró, J. M. (1989). La medida de la satisfacción laboral en contextos organizacionales: el cuestionario de satisfacción S20/23. Psicologema, 5, 59-74.

Misener, T., Haddock, K., Gleaton, J. U. \& Ajamieh, A. R. (1996). Toward and international measure of job satisfaction. Nursing Research, $45,87-91$.

Morana, C. (1987). Employee satisfaction: a key to patient satisfaction. Perioperative Nursing Quarterly, 3(1), 33-37.

Moreno, B., Oliver, C. y Aragoneses, A. (1991). El Burnout: una forma específica de estrés laboral. En G. Buela y V. E. Caballo (Eds.). Manual de psicología clínica aplicada (pp. 271-284). Madrid: Siglo XXI.

Peiró, J. M. (2004b). El sistema de trabajo y sus implicaciones para la prevención de los riesgos psicosociales en el trabajo. Universitas Psychologica, 3(2), 179-186.
Peiró, J. M. y Rodríguez, I. (2008). Estrés laboral, liderazgo y salud organizacional. Papeles del Psicólogo, 29(1), 68-82.

Peiró J. M., Silla, I., Sanz, T., Rodríguez, J. y García, J. L. (2004a). Satisfacción laboral de los profesionales de atención primaria. Psiquis, 25, 129-140.

Pitcher, P. (1997). The drama of Leadership. New York: John Wiley and Sons.

Pitcher, P. (1999). Artists, craftsmen \& technocrats. Training \& Development, 30-33.

Porter, L. \& Lawler, E. (1968). Managerial attitudes and performance. New York: Irwin Dorsey.

Regina, V., Cardoso, M. C. \& Oliveira, M. (2010). Burnout and stress among nurses in an university tertiary hospital. Revista LatinoAméricana de Enfermagem, 8(6), 1084-1091.

Smith, P., Kendall, L. M. \& Hulin, C. L. (1975). The measurement of satisfaction in work and retirement. Chicago: Rand McNally.

Sosik, J. J. \& Godshalk, V. M. (2000). Leadership styles, mentoring functions received and jobrelated stress: a conceptual model and preliminary results. Journal of Organizational Behavior, 21(4), 365-390.

Topa, G., Fernández, I. y Palací, F. (2006). Identidad social, Burnout y satisfacción laboral: estudio empírico basado en el modelo de la categorización del yo. Revista de Psicología Social, 21(2), 115-126.

Upenieks, V. V. (2003). The interrelationship between organizational characteristics of magnet hospitals, nursing leadership, and nursing job satisfaction. The Health Care Manager, 22(2), 83-98.

Zalon, M. L. (1995). Pain management instruction in nursing curricula. Journal of Nursing Education, 34, 2622-2627. 